\title{
Listening to the Universe with Gravitational Waves
}

\section{Karsten Danzmann ${ }^{1}$}

AEI Hannover: Max Planck Institute for Gravitational Physics and Leibniz Universität Hannover Callinstr. 38, 30167 Hannover, Germany

E-mail: karsten.danzmann@aei.mpg.de

Gravitational waves have been predicted by Albert Einstein more than 90 years ago as a consequence of his theory of general relativity. Several km-size gravitational wave detectors have now gone into operation on the ground to observe signals at frequencies from a few $\mathrm{Hz}$ to a few kHz. In 2020, LISA, a space-based detector, will open the low-frequency window from 0.1 $\mathrm{mHz}$ to $0.1 \mathrm{~Hz}$.

European Physical Society Europhysics Conference on High Energy Physics EPS-HEP 2009, Krakow , Poland

July 16-22, 2009

Speaker 


\section{Introduction}

Gravitational waves have been predicted by Albert Einstein more than 90 years ago as a consequence of his theory of general relativity. Several km-size gravitational wave detectors have now gone into operation on the ground to observe signals at frequencies from a few $\mathrm{Hz}$ to a few kHz. In 2020, LISA, a space-based detector, will open the low-frequency window from $0.1 \mathrm{mHz}$ to $0.1 \mathrm{~Hz}$. Gravitational wave detecors will give us insights into the dark side of the universe that we cannot obtain in any other way.

\section{Ground-based detectors}

Because of the unshieldable background from Newtonian gravitation, only the frequency range above $1 \mathrm{~Hz}$ is accessible from ground. Large laser interferometers operate here and are sensitive from a few $\mathrm{Hz}$ up to a few $\mathrm{kHz}$. In this frequency range, we expect short signals from stellar mass type sources, such as the coalescences of compact binary stars made from neutron stars and/or stellar mass black holes. Supernova core collapse events will also emit in this range. Quasi-monochromatic signals in this audio frequency range will be emitted by rotating asymmetric neutron stars.

\subsection{First generation detectors}

First generation detectors comprise LIGO in the US (4 km armlength, 2 sites at Hanford and Livingston), Virgo in Italy (French-Italian project, $3 \mathrm{~km}$ armlength, 1 site in Cascina near Pisa), GEO600 (German-British project, 0.6 km armlength, in Ruthe near Hannover), TAMA (Japan, $0.3 \mathrm{~km}$ armlength, in Tokyo). The LIGO detectors, with participation from GEO and Virgo, have finished their first year-long data taking run S5 and have just started the S6 run, to be followed in 2011 by upgrading of the hardware.

\subsection{Second generation detectors}

After major hardware upgrades, the Advanced LIGO and Advanced Virgo detectors will go online again for data taking in 2015. It is expected that the sensitivity will be tenfold improved in amplitude for this second generation. The expected event rates will be between several per month and many per day.

\subsection{Third generation detectors}

In 2008, the European Community started funding a Design Study for a Third Generation Gravitational Wave Observatory, called the Einstein Gravitational Wave Telescope, or in short, ET. All European gravitational wave groups are involved in this Design Study. The expected sensitivity will be an order of magnitude better than even the second generation detectors, 
making high-resolution, high-event rate Gravitational Wave Astronomy a reality. Construction could begin before the end of the decade.

\section{Space-based detectors}

The frequency range from $0.03 \mathrm{mHz}$ to $0.1 \mathrm{~Hz}$ contains both the most secure and the most spectacular sources of gravitational waves. These frequencies are only accessible from space. Mergers of supermassive black holes will be detectable with large signal-to-noise ratios wherever they occur in the universe, serving as standard candles and giving the absolute luminosity distance over cosmological scales with high precision and accuracy.

\subsection{LISA}

LISA is a planned joint ESA/NASA space mission for the observation of low-frequency gravitational waves. It comprises a cluster of three spacecraft at the corners of an equilateral triangle with 5 million $\mathrm{km}$ armlength in heliocentric orbit 20 degrees behind the earth. LISA has been under study since 1992 and the baseline concept has been basically unchanged since 1997. Since 2005, LISA is in the Misison Formulation Phase of its mission lifecycle. It is an L1 launch candidate in the ESAS Cosmic Vision program and has been a flagship mission in the NASA Beyond Einstein mission suite. The foreseen launch date is in 2020.

\subsection{LISA Pathfinder}

LISA Pathfinder is the technology demonstrator mission for LISA. It is an ESA mission with payload contributions from NASA. The technology tested on Pathfinder comprises lasers, interferometry, gravitational reference sensors, micro-Newton thrusters, and drag-free control. Allo $\mathrm{f}$ it is nominally of the LISA design, retiring the remaining development risk for LISA considerably. LISA Pathfinder is currently in the implementation phase and flight hardware is being built and delivered. The launch is planned for summer of 2012.

\section{Conclusion}

Multi-wavelength gravitational wave astronomy is rapidly becoming a reality, with first ground based detections highly likely by 2015 after Advanced LIGO and Advanced VIRGO go online, and high SNR observations over cosmological distances becoming routine with LISA after 2020.

\section{References}

[1] GEO600: geo600.aei.mpg.de

[2] LIGO: www.ligo.caltech.edu

[3] VIRGO: www.virgo.infn.it

[4] LISA: www.lisa.aei-hannover.de

[5] LISA Pathfinder: sci.esa.int/science-e/www/area/index.cfm?fareaid=40 